\title{
Trasplante renal en el receptor del virus de la hepatitis $\mathrm{C}$ positivo
}

\author{
Kidney transplantation in hepatitis $C$ virus-positive recipients
}

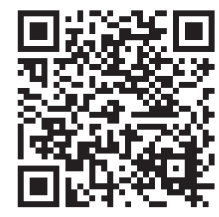

\author{
Ignacio García-Juárez* \\ * Departamento de Gastroenterología. Instituto Nacional de Ciencias Médicas y Nutrición «Salvador Zubirán».
}

\section{DETECCIÓN DEL VIRUS DE LA HEPATITIS C}

1. El tamizaje de virus de la hepatitis (VHC) debe ser establecido de acuerdo con la epidemiología local. Éste se basa en pruebas de inmunoensayo (ELI$\mathrm{SA}$ ) en plasma o suero, por ejemplo, la prueba de tamizaje de primera línea.

2. Si la prueba es positiva (anticuerpos del VHC), se debe solicitar una prueba confirmatoria de ARN del virus de la hepatitis con un límite inferior de $15 \mathrm{UI} /$ $\mathrm{mL}$.

3. El tamizaje del VHC se debe ofrecer con medidas de prevención, cuidados y tratamiento.

La infección por virus de la hepatitis $\mathrm{C}(\mathrm{VHC})$ es, por lo general, asintomática. Para su tamizaje, se requiere de una prueba que evalúe la presencia de anticuerpos del VHC. Para el caso de la enfermedad renal, se ha evaluado el circuito interno de la máquina de hemodiálisis, y se considera que es un contribuidor menor para la transmisión del VHC en pacientes con hemodiálisis. ${ }^{1}$ Uno de los mayores factores para su transmisión se ha asociado con la contaminación cruzada del material y de las superficies por la falta de adherencia a los procedimientos de control de infecciones. ${ }^{1}$ Por ello, se recomienda realizar una prueba para $\mathrm{VHC}$ en aquellos pacientes con reciente diagnóstico de enfermedad renal. ${ }^{2,3}$ Asimismo, se recomienda realizar una prueba en aquellos pacientes que empiezan la hemodiálisis o en aquéllos que se transfieren entre centros de hemodiálisis; ${ }^{2}$ además, se sugiere complementar con serología para hepatitis $\mathrm{B}$ y evaluación de alanino aminotransferasa (ALT). ${ }^{1}$

Se ha recomendado que los pacientes en diálisis con una serología negativa para VHC tengan un retamizaje cada seis meses, ${ }^{1}$ aunque según la Norma Oficial Mexicana NOM-003-SSA3-2019, para la práctica de la hemodiálisis, es necesario realizarlo cada cuatro meses.

Se debe recordar que en pacientes con una enfermedad renal terminal, la serología para VHC es insuficiente. ${ }^{1}$ El diagnóstico se basa en la presencia de viremia en sangre o plasma por un método cuantitativo cuyo límite inferior de detección es $<15$ unidades internacionales $(\mathrm{IU}) / \mathrm{mL} .^{3}$

Virus de hepatitis C y enfermedad renal crónica

1. La infección por VHC es una causa importante de morbilidad y mortalidad en los pacientes con enfermedad renal crónica terminal y hemodiálisis.

La infección por VHC se ha asociado con altas tasas de morbilidad y mortalidad en enfermedades del hígado, y de un 40 a $70 \%$ de los casos puede estar acompañado de manifestaciones extrahepáticas, como enfermedades autoinmunes, metabólicas, renales, cardiovasculares, de sistema nervioso central, entre otras. ${ }^{2}$

La enfermedad renal es una manifestación extrahepática común. Esta enfermedad puede contribuir no sólo a un deterioro renal progresivo, sino que 
también puede llevar a una enfermedad renal crónica terminal (ERCT). ${ }^{4} \mathrm{~A}$ su vez, la infección por VHC incrementa la morbilidad y las tasas de mortalidad en pacientes con hemodiálisis y en receptores de trasplante renal. ${ }^{2,4}$

En el caso de la enfermedad renal crónica (ERC) y el $\mathrm{VHC}$, se requieren diferentes consideraciones en los espectros de presentación, ya que, en algunos grupos, la función renal podría mejorar luego del tratamiento con antivirales de acción directa (AAD). ${ }^{3}$ En este tratamiento se incluyen a los pacientes con ERC en estadio 4 (función severamente reducida y TFG = $15-29 \mathrm{~mL} / \mathrm{min} / 1.73 \mathrm{~m}^{2}$ ) o en aquellos pacientes con ERCT (con TFG $<15 \mathrm{~mL} / \mathrm{min} / 1.73 \mathrm{~m}^{2}$ o diálisis); pacientes luego de un trasplante renal; pacientes con falla renal y cirrosis; pacientes postrasplantados hepáticos con lesión renal por inhibidores de calcineurina, y pacientes con crioglobulinemia mixta esencial con lesión renal. ${ }^{2,3}$

Con el advenimiento de los AAD, el tratamiento poco a poco ha llegado a tasas de respuesta viral sostenida (RVS) > 98; esto ha provocado una revolución en el tratamiento de infección por $\mathrm{VHC},{ }^{4}$ convirtiéndola en una enfermedad con un potencial de curación y de erradicación.

Se considera que la prevalencia de infección por VHC en pacientes en prediálisis es del $7.6 \%,{ }^{4}$ y ésta se incrementa con el estadio de la enfermedad renal, incluso se ha postulado que existe una correlación entre VHC y la función renal, y que esta infección puede promover la presencia de ERC y, finalmente, llevar a ERCT. ${ }^{4-6}$

En el caso de los pacientes en diálisis, la prevalencia de infección por VHC puede ser de 4-13.5\%;,4,6,7 sin embargo, según la región, ésta puede cambiar desde un 2.6 a un $22.9 \%,{ }^{4} \mathrm{e}$ incluso puede variar en pacientes con hemodiálisis (HD) de pacientes con diálisis peritoneal (de 7.9 a $2.0 \%$, respectivamente). ${ }^{4}$ De la misma manera, a mayor tiempo en $\mathrm{HD}$, existe un incremento en la prevalencia del VHC (58\% en > 20 años). ${ }^{4}$

La prevalencia del VHC en pacientes receptores de trasplante renal (TR) es de 6-46\%; muchos de ellos se infectan durante la diálisis más que después de haber recibido el trasplante. ${ }^{4}$

Los pacientes trasplantados requieren el uso de inmunosupresores. Éste es un factor importante para el desarrollo de una condición conocida como diabetes de reciente inicio luego del trasplante (NODAT), que a su vez puede contribuir a lesión renal de forma posterior. ${ }^{8}$ Se ha visto que la prevalencia de NODAT en receptores de TR puede ser de hasta $25.6 \%$ en infectados por VHC en comparación con aquéllos sin la infección con $15.4 \%,{ }^{4}$ y el OR se ha estimado en 3.97 (IC 95\% 1.83-8.61).

\section{Evaluación de la función hepática en pacientes con VHC y enfermedad renal crónica}

1. La severidad de la función hepática debe ser evaluada antes del inicio de la terapia con AAD mediante métodos no invasivos (marcadores serológicos como APRI o FIB-4 o elastografía). Actualmente, la biopsia hepática sólo debe ser considerada en caso de sospecha de enfermedad mixta en el hígado.

2. Los pacientes con fibrosis avanzada (F3-F4) o cirrosis descompensada pueden requerir ajustes en su esquema de tratamiento. Estos pacientes requieren una evaluación de la hipertensión portal y seguimiento cada seis meses con ultrasonidos hepático para vigilar si se presenta carcinoma hepatocelular.

En pacientes con infección por virus de hepatitis $\mathrm{C}$, es importante determinar qué grado de fibrosis hepática presentan al momento del diagnóstico. Por años, la biopsia hepática se había considerado como el estándar de referencia, ${ }^{9}$ sin embargo, es un método invasivo y cuenta con algunas limitaciones en su certeza diagnóstica, por ejemplo, el error de muestreo (el tamaño de la muestra tiene que ser de $1 / 50,000$ del total de la masa hepática, se requiere por lo menos $15 \mathrm{~mm}$, con una evaluación optima con $25 \mathrm{~mm}$ de longitud $^{10}$ y la posibilidad de variabilidad inter e intraobservador). ${ }^{9,11}$

Tabla 1: Esquemas propuestos para tratamiento de pacientes con enfermedad renal crónica estadio 405 .

\begin{tabular}{lcccc} 
Clearence creatinina & $15-29 \mathrm{~mL} / \mathrm{min} / 1.73 \mathrm{~m}^{2}$ (severo) & $<15 \mathrm{~mL} / \mathrm{min} / 1.73 \mathrm{~m}^{2}$ (ERCT) & Genotipo & Tiempo \\
\hline Grazoprevir/elbasvir & $100 \mathrm{mg} / 50 \mathrm{mg}$ & $100 \mathrm{mg} / 50 \mathrm{mg}$ & 12 semanas \\
Glecaprevir/pibrentasvir & $300 \mathrm{mg} / 120 \mathrm{mg}$ & $300 \mathrm{mg} / 120 \mathrm{mg}$ & $1,2,3,4,5,6$ & $8 \mathrm{a} 16 \mathrm{semanas}$ \\
\hline
\end{tabular}

ERCT = enfermedad renal crónica terminal. 
Para la evaluación histológica de la fibrosis se utilizan diferentes escalas, como la de Knodell, Ishak y la del grupo colaborativo METAVIR. Es importante identificar a los pacientes con un grado de fibrosis avanzado (METAVIR F3 o F4), ya que el pronóstico postratamiento depende del estadio de la fibrosis. ${ }^{12}$

Hoy en día, disponemos de dos métodos no invasivos diferentes para la determinación de la fibrosis: 1 . Biológico: basado en la cuantificación de biomarcadores en muestras de suero (indican varios parámetros clínicos y serológicos no específicamente de hígado, los cuales han sido asociados con el estadio de fibrosis). En este método existen varios algoritmos comerciales. 2. El físico: basado en la medición de la rigidez hepática (evaluación intrínseca de las propiedades del parénquima). Este método comprende la elastografía por FibroScan o por resonancia magnética. ${ }^{11}$

Aquellos pacientes con cirrosis hepática (F4) necesitan una evaluación para hipertensión portal (seguimiento de várices esofágicas). Se debe considerar la posibilidad de utilizar esquemas de tratamiento con diferente duración. Además, cabe considerar que los pacientes van a requerir seguimiento postratamiento cada seis meses con ultrasonido hepático para detectar carcinoma hepatocelular (HCC). ${ }^{3}$

Tratamiento del virus de la hepatitis $C$ en la lista de espera de trasplante renal

1. Los pacientes con enfermedad renal terminal y con tasa de filtración glomerular (TFG) $<30 \mathrm{~mL} /$ $\mathrm{min} / 1.73 \mathrm{~m}^{2}$ y los pacientes en hemodiálisis requieren un manejo multidisciplinario.

2. Los pacientes con enfermedad renal terminal y con TFG $<30 \mathrm{~mL} / \mathrm{min} / 1.73 \mathrm{~m}^{2}$ o en hemodiálisis deben ser tratados con un esquema pangenotípico (1 a 6) como glecaprevir/pibrentasvir durante 8 semanas; en pacientes no cirróticos y con cirrosis se compensará durante 12 semanas. También se puede utilizar el esquema de grazoprevir/elbasvir durante 12 semanas para genotipo 1 y 4 .

3. En pacientes con TFG $<30 \mathrm{~mL} / \mathrm{min} / 1.73 \mathrm{~m}^{2}$, el sofosbuvir debe ser utilizado sólo si no existe un tratamiento alternativo aprobado para su uso.

Para el tratamiento en este grupo de pacientes, la seguridad de sofosbuvir se debe evaluar en cada caso. El sofosbuvir, al ser eliminado por vía renal, no cuenta con la aprobación de uso para los pacientes en enfermedad renal terminal estadio 4 o $5 \mathrm{e}$, incluso, en los que requieren hemodiálisis. ${ }^{3}$
El estudio EXPEDITION-4 es un ensayo clínico fase 3 en el que se utilizó glecaprevir/pibrentasvir durante 12 semanas; éste involucró a 104 pacientes en estadio 4 y 5 de la enfermedad renal de los cuales el $82 \%$ estuvo en diálisis y $19 \%$ fue de pacientes cirróti$\cos ^{3}$ En este estudio, la tasa de RVS fue de $98 \%$ en el análisis de intención a tratar, y de $100 \%$ en el análisis modificado (se tomó en consideración que las fallas se debieran a muerte y a la pérdida de seguimiento). Incluso, la tasa en otros estudios fase II y fase III para estadio 4 y 5 fue del $98 \%{ }^{2}$

En el estudio EXPEDITION-5, el uso de la combinación de glecaprevir y pibrentasvir presentaron RVS similares de ocho semanas en 84 pacientes, de 12 semanas en 13 pacientes y de 16 semanas en cuatro pacientes. ${ }^{2}$

4. Los pacientes sin indicación de trasplante renal y con una carga viral $<800.000 \mathrm{IU} / \mathrm{mL}$ pueden recibir un tratamiento de 12 semanas de grazoprevir/ elbasvir (aplica a genotipo 1a, 1b y 4).

En el ensayo C-SURFER, 122 pacientes infectados por el virus de la hepatitis $\mathrm{C}$ (VHC) genotipo 1, en estadio 4 o 5 de la enfermedad renal y con un $75 \%$ en hemodiálisis recibieron tratamiento durante 12 semanas con ribavirina. La RVS a la semana 12 fue de $94 \%$ con una falla virológica. En el análisis por protocolo la RVS fue de $99 \% .^{2}$ El efecto adverso más común fue la cefalea, náusea y fatiga. En estudios de vida real se ha observado un RVS de $96 \% .^{3}$ La vía de excreción del fármaco principalmente ocurre a través de las heces, por lo que no se han mostrado datos de toxicidad renal. ${ }^{2}$

5. Los pacientes deben ser tratados antes o después del trasplante si su expectativa de vida excede un año. Los pacientes en lista de espera deben ser tratados con esquemas y duración que se basen en la severidad de la enfermedad hepática y en los tratamientos previos de VHC.

La decisión para tratar el VHC, en relación con el tiempo para el trasplante renal, debe considerar el tipo de donador (vivo o cadavérico), el tiempo de espera, según el tipo de sangre del donador, las políticas específicas del centro para uso de donadores infectados de VHC positivos ( $\mathrm{D}+/ \mathrm{R}+$ ), el genotipo y la severidad de la fibrosis hepática. ${ }^{3,13}$

Recibir un riñón de un donador VHC positivo incrementa la oportunidad de recibir un trasplante, por lo que el tratamiento para VHC se puede realizar después de este mismo, ${ }^{3}$ sin embargo, es importante 
Tabla 2: Esquema de tratamiento para pacientes posterior al trasplante renal.

\begin{tabular}{lccc}
\multicolumn{1}{r}{ Naive/experimentados } & Tratamiento & Dosis (mg/mg) & Tiempo \\
\hline Genotipo 104 & Sofosbuvir/ledipasvir 4 & $400 / 90$ & 12 semanas \\
Con/sin cirrosis compensada & Glecaprevir/pibrentasvir & $300 / 120$ & $8 \mathrm{a} 16$ semanas \\
Genotipo 1, 2, 3, 4, 506 & Glecaprevir/pibrentasvir & $300 / 120$ & 12 semanas \\
Con/sin cirrosis compensada & Daclatasvir/sofosbuvir/ribavirina & $60 / 400 / 600$ & 12 semanas \\
\hline
\end{tabular}

contar con la experiencia clínica necesaria para determinar las potenciales interacciones con los fármacos inmunosupresores, así como la posibilidad de infecciones por múltiples cepas virales. ${ }^{9,14}$

Entre las estrategias conocidas está: dar una dosis de grazoprevir de $100 \mathrm{mg}$ junto con elbasvir de $50 \mathrm{mg}$ antes del trasplante y mantener el tratamiento durante 12 semanas en genotipo 1. Resultados han demostrado una RVS de $100 \% .{ }^{15}$

La infección por VHC en receptores de trasplante renal puede estar asociada con un incremento en la progresión de la fibrosis hepática. La mayoría de cohortes de pacientes con trasplante renal ha demostrado que la positividad por VHC en el seguimiento se asocia con falla del injerto y reducción de la sobrevida, más aún en pacientes con cirrosis si no es tratado el VHC. ${ }^{3,6}$

\section{TRASPLANTE RENAL}

1. Los pacientes postrasplantados renales pueden ser tratados con un esquema que se base en el tratamiento de sofosbuvir/ledipasvir o glecaprevir/ pibrentasvir durante 12 semanas.

2. En caso de tener una función renal $<30 \mathrm{~mL} /$ $\mathrm{min} / 1.73 \mathrm{~m}^{2}$ posterior al trasplante renal, se puede utilizar un esquema de glecaprevir/pibrentasvir durante 12 semanas.

3. Se debe ajustar la inmunosupresión según sea necesario y se deben verificar las interacciones con otros medicamentos.

En el caso de los pacientes postrasplantados, la interacción con inmunosupresores ha sido una preocupación. El sofosbuvir se puede administrar, ya que no presenta interacciones con inhibidores de calcineurina, micofenolato mofetil o azatioprina. ${ }^{6} \mathrm{La}$ combinación de glecaprevir y pibrentasvir con ciclosporina puede incrementar los niveles cinco veces, y con tacrolimus, 1.45 veces. ${ }^{6}$

El uso de sofosbuvir/ledipasvir ha demostrado RVS de $100 \%$ para genotipo 1 y 4 con esquemas de 12 o
24 semanas. En el estudio de Prasad y colaboradores, ${ }^{16}$ la tasa de respuesta fue de $100 \%$ a las 12 y 24 semanas de seguimiento con esquemas de sofosbuvir/ledipasvir o daclatasvir, sin embargo, apareció una disminución de la concentración de los niveles de tacrolimus, por lo que se debe realizar un ajuste en la dosis de inmunosupresores.

\section{Seguimiento después de la RVS}

1. En todos los pacientes con fibrosis $(\leq F 2)$ y en hemodiálisis, una vez que se corroboré la RVS, debe seguirse monitoreando la reacción en cadena de la polimerasa (PCR) de VHC cada año.

2. En todos los pacientes con fibrosis ( $\geq F 3$ ) y en hemodiálisis una vez que se corrobore la RVS debe seguirse monitoreando la PCR de VHC cada año y el ultrasonido hepática cada seis meses.

3. Paciente con RVS y de alto riesgo de reinfección (uso de drogas inyectables o hombres que tienen sexo con hombres) debe seguirse monitoreando la PCR de VHC cada año.

Se debe considerar que, una vez que se ha logrado la RVS en pacientes con hemodiálisis o aquéllos con alto riesgo de reinfección, es importante el seguimiento con PCR de VHC de forma anual; así mismo, todos aquéllos con fibrosis avanzada (F3-F4) deben seguir las guías internacionales para detección de carcinoma hepatocelular. ${ }^{2,17}$

\section{Uso de órganos VHC positivos}

1. Los donantes de órganos con anticuerpo y ARN positivos para VHC pueden ser sujetos de trasplante en receptores ARN positivos para VHC. Respecto del uso de órganos con anticuerpo y ARN positivo para VHC en receptores VHC negativo, se deberá considerar en cada Comité de Trasplante según su disponibilidad y con previo consentimiento informado del receptor. 
2. En caso de uso de órganos con anticuerpo y ARN del virus de la hepatitis para receptores VHC negativos, se deberá iniciar tratamiento con AAD a la brevedad.

Hasta el momento no existen suficientes órganos para suplir todas las demandas de trasplante. Esto ha generado una mayor presión para obtener soluciones y considerar la utilización de donadores con infecciones que tengan riesgo de transmitir VHC. ${ }^{5,13,14,18}$ Además, el tiempo desde el inicio de la diálisis al trasplante renal puede ser de 3.96 años en países como Canadá o Estados Unidos, mientras que en México este tiempo puede ser mayor. Utilizar órganos con anticuerpos positivos para VHC (Ac$\mathrm{VHC}+$ ), incluyendo aquéllos con viremia positiva, puede incrementar el acceso a trasplante ${ }^{3}$ y puede ser costo-efectiva si por lo menos hay una reducción de más de cuatro años en el tiempo de espera del trasplante renal. ${ }^{14}$

Debido a la ola de tratamientos con AAD, la población de donación puede presentar un anticuerpo positivo para VHC, sin embargo, la viremia o RNA-VHC debe ser negativa. Esta distinción es importante, ya que la transmisión es inusual. Si bien se han reportado casos raros de transmisión, esto, probablemente, se debe a casos de infección aguda en pacientes de alto riesgo..$^{3,13}$

A partir de 2015, en Estados Unidos, la prueba de amplificación nuclear (NAT) para VHC se debía realizar y reportar junto al estado serológico de la enfermedad para todos los donadores de VHC. ${ }^{14}$ Esta conducta finalmente permitió realizar una distinción entre los diferentes riesgos asociados con donadores $\mathrm{VHC}$ positivos (Ac-VHC +) pero con NAT negativo (NAT -), los cuales se consideran con un riesgo mínimo de transmisión. ${ }^{13,19,20}$

\section{REFERENCIAS}

1. Burra P, Rodríguez-Castro KI, Marchini F, Bonfante L, Furian L, Ferrarese $A$ et al. Hepatitis $C$ virus infection in end-stage renal disease and kidney transplantation. Transpl Int. 2014; 27: 877-891.

2. Pol $S$, Parlati $L$, Jadoul $M$. Hepatitis $C$ virus and the kidney. Nat Rev Nephrol. 2019; 15: 73-86.

3. Pawlotsky JM, Negro F, Aghemo A, Berenguer M, Dalgard $O$, Dusheiko $G$ et al. EASL recommendations on treatment of hepatitis c 2018. J Hepatol. 2018; 69: 461-511.

4. Kim SM, Song IH. Hepatitis $\mathrm{C}$ virus infection in chronic kidney disease: paradigm shift in management. Korean $\mathrm{J}$ Intern Med. 2018; 33: 670-678.

5. Joglekar K, Eason JD, Molnar MZ. Do we really need more evidence to use hepatitis $\mathrm{C}$ positive donor kidney more liberally? Clin Kidney J. 2017; 10: 560-563.
6. Salvadori M, Tsalouchos A. Hepatitis $C$ and renal transplantation in era of new antiviral agents. World $\mathrm{J}$ Transplant. 2018; 8: 84-96.

7. Cohen JB, Eddinger KC, Shelton B, Locke JE, Forde KA, Sawinski D. Effect of kidney donor hepatitis $C$ virus serostatus on renal transplant recipient and allograft outcomes. Clin Kidney J. 2017; 10: 564-572.

8. Fernandes AR, Laranjinha IJ, Birne R, Matias P, Jorge C, Adragão $T$ et al. HCV-infected renal transplant recipients: our experience before the availability of new antiviral drugs. Int $\mathrm{J}$ Organ Transplant Med. 2017; 8 (2): 104-109.

9. Conti F, Serra C, Vukotic R, Felicani C, Mazzotta E, Gitto $S$ et al. Assessment of liver fibrosis with elastography point quantification vs other noninvasive methods. Clin Gastroenterol Hepatol. 2019; 17: 510-517.

10. Buckley AJ, Thomas EL, Lessan N, Trovato FM, Trovato GM, Taylor-Robinson SD. Non-alcoholic fatty liver disease: Relationship with cardiovascular risk markers and clinical endpoints. Diabetes Res Clin Pract. 2018; 144: 144-152.

11. EASL-ALEH Clinical Practice Guidelines: Non-invasive tests for evaluation of liver disease severity and prognosis. J Hepatol. 2015; 63: 237-264.

12. Younossi Z, Anstee QM, Marietti M, Hardy T, Henry L, Eslam M et al. Global burden of NAFLD and NASH: trends, predictions, risk factors and prevention. Nat Rev Gastroenterol Hepatol. 2017; 15: 11-20.

13. Sawinski D, Wyatt CM, Locke JE. Expanding the use of hepatitis C-viremic kidney donors. Kidney Int. 2017; 92: 1031-1033.

14. Kadatz M, Klarenbach S, Gill J, Gill JS. Cost-effectiveness of using kidneys from hepatitis $C$ nucleic acid test-positive donors for transplantation in hepatitis C-negative recipients. Am J Transplant. 2018; 18: 2457-2464.

15. Durand CM, Bowring MG, Brown DM, Chattergoon MA, Massaccesi G, Bair N et al. Direct-acting antiviral prophylaxis in kidney transplantation from hepatitis $C$ virus-infected donors to noninfected recipients: an open-label nonrandomized trial. Ann Intern Med. 2018; 168: 533.

16. Prasad N, Patel M, Pandey A, Jaiswal A, Bhadauria D, Kaul A et al. Direct-acting antiviral agents in hepatitis $C$ virus-infected renal allograft recipients: treatment and outcome experience from single center. Indian J Nephrol. 2018; 28: 220.

17. Aiza Haddad I, Ballesteros Amozurrutia A, Borjas Almaguer OD, Castillo Barradas M, Castro Narro G, Chávez Tapia N et al. Consenso Mexicano para el tratamiento de la hepatitis C. Revista de Gastroenterología de México. 2018; 83 (3): 275-324.

18. Gupta G, Zhang Y, Carroll NV, Sterling RK. Cost-effectiveness of hepatitis C-positive donor kidney transplantation for hepatitis C-negative recipients with concomitant direct-acting antiviral therapy. Am J Transplant. 2018; 18: 2496-505.

19. Sibulesky L, Kling CE, Blosser C, Johnson CK, Limaye AP, Bakthavatsalam $\mathrm{R}$ et al. Are we underestimating the quality of aviremic hepatitis C-positive kidneys? Time to reconsider. Am J Transplant. 2018; 18: 2465-2472.

20. Gallegos-Orozco JF, Kim R, Thiesset HF, Hatch J, Lynch K, Chaly $\mathrm{Jr} T$ et al. Early results of pilot study using hepatitis $\mathrm{C}$ virus (HCV) positive kidneys to transplant HCV infected patients with end-stage renal disease allowing for successful interferonfree direct acting antiviral therapy after transplantation. Cureus. 2016; 8 (11): e890.

Correspondencia:

Dr. Ignacio García-Juárez

E-mail: drinter77@gmail.com 